PAPER • OPEN ACCESS

\title{
Passive autonomy: hygromorphic rotational actuators
}

To cite this article: Sebastiaan Joosten et al 2021 Smart Mater. Struct. 30025008

View the article online for updates and enhancements. 


\title{
Passive autonomy: hygromorphic rotational actuators
}

\author{
Sebastiaan Joosten ${ }^{1}\left(\mathbb{D}\right.$, Giuseppe Radaelli $^{2}(\mathbb{D})$ and Heike Vallery ${ }^{1,3}(\mathbb{D})$ \\ ${ }^{1}$ Department of BioMechanical Engineering, Delft University of Technology, Delft, The Netherlands \\ ${ }^{2}$ Department of Precision and Microsystems Engineering, Delft University of Technology, Delft, The \\ Netherlands \\ ${ }^{3}$ Department of Rehabilitation, Erasmus MC, Rotterdam, The Netherlands \\ E-mail: S.T.Joosten@ @tudent.tudelft.nl and G.Radaelli@tudelft.nl
}

Received 4 August 2020, revised 17 November 2020

Accepted for publication 30 November 2020

Published 21 December 2020

\begin{abstract}
Inspired by phenomena in the plant world, a meteoro-sensitive rotational actuator is developed. The design uses a hygro-active shell, whose water-based swelling is restricted at selective locations to form a helicoid structure. The influence of geometrical parameters on the performance is investigated using a numerical analysis of various geometries, by looking at resulting rotation and torque during this rotation. Prototypes are built of five key geometries in the design space, to validate the simulations and to investigate the behaviour of the design experimentally. These prototypes are submerged in water to investigate their deformation, after which they are placed in a torsion machine to investigate the torque during rotation. The experiments result in similar rotations and torques as the simulations. The designed Hygromorphic Rotational Actuator is capable of passively rotating its own structure, thereby expanding the possibilities of engineers and designers when designing passive autonomous systems.
\end{abstract}

Supplementary material for this article is available online

Keywords: hygromorphic, multi-stable, zero-moment, meteoro-sensitive, actuator, adaptive structure

(Some figures may appear in colour only in the online journal)

\section{Introduction}

In engineering, swelling and shrinking of a material in response to absorbing or desorbing water is generally something to avoid. Plants, however, often use this phenomenon to their advantage [1-3].

Hygroscopy is frequently used by plants as basic movement initiator to create a passive motion. Fibrous tissue swells because it equilibrates its water content with the ambient

Original Content from this work may be used under the terms of the Creative Commons Attribution 4.0 licence. Any further distribution of this work must maintain attribution to the author(s) and the title of the work, journal citation and DOI. humidity [4]. This swelling is selectively resisted to amplify the movement [5], for instance by a layer of non-hygroscopic material. The resulting bilayer structure will bend when exposed to water [6]. Well-known examples are the opening of seedpods such as pinecones [7] or the movement of wheat awns [2].

Other amplification mechanisms that plants use to amplify a basic movement initiator, are based upon what humans would call failure mechanisms. Various seed catapults rely on fracture to release stored energy, shooting their seeds away $[1,8]$. Flower opening often relies on differential elongation of the edge with respect to the midrib, causing a snap-through buckling $[9,10]$.

The combination of water-driven swelling or shrinking of structures with a selective restriction of this swelling or shrinking enables the autonomous movement of plant structures 
without costing energy. Sensing, actuating and moving happens within the same structure, saving space and increasing efficiency $[5,11]$.

In some disciplines, methodologies are developed to incorporate natural working principles in the design process $[12,13]$. Engineers and designers are gradually adopting these hygromorphic shell structures in their designs [14-16]. Most notably, hygro-active building envelopes are being developed to passively regulate the shading and ventilation of buildings, in response to the surrounding humidity [17-19]. Hygromorphic bilayers are even being used for the locomotion of small hygro-active robots [20].

These examples show that passive hygromorphic actuators can benefit various engineering fields. They move completely autonomous based on environmental stimuli and do not require a supply of energy, making them suitable for interesting applications. However, the majority of existing hygromorphic shell actuators are based on bending or translation [3, 14-16, 21]. Although some twisting hygro-active structures have been developed, they are built out of composite multilayer segments that deform simultaneously and as a result their rotation can not be precisely controlled [22].

In order to expand and improve the possibilities of these passive autonomous structures, this research focusses on the development of a hygroscopic shell that rotates with increasing environmental humidity. Its rotation can be controlled by careful selection of the main dimensional parameters.

Two working principles found in the plant world are combined. Hygroscopic swelling is used as a basic movement initiator to start the motion. Selective restriction of this translational swelling is used to cause differential edge elongation. This elongation serves as the amplification mechanism, which transfers the small swelling to a larger rotation.

The design is analysed by building a finite element model based on an isogeometric framework. The influence of the main geometrical parameters on the resulting rotation and torque is investigated. Finally, a set of prototypes is fabricated to validate the behaviour of the design experimentally and to verify the simulated model.

\section{Methods}

\subsection{Conceptual design}

To generate a hygromorphic rotation with a shell structure, two of the aforementioned working principles from the plant world are combined (see figure 1). The presented hygromorphic rotational actuator (HRA) consists of a rectangular plate of orthotropic hygroscopic material. When exposed to water, this material only extends in the vertical direction. A common example of a material with these properties is wood [23].

This hygroscopic expansion is selectively resisted by applying constraints. While in a bilayer system the constraint consists of a complete second non-hygro-responsive shell attached to the first one [2], here only a constraint restricting expansion across the vertical centreline of the shell is applied. Furthermore, both the top and bottom horizontal edges are forced to maintain a right angle with this centreline. Their movement is

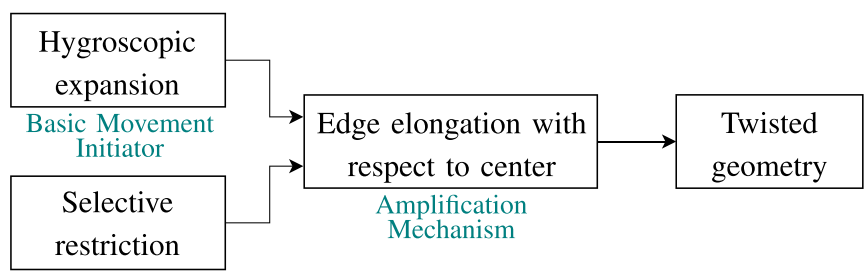

Figure 1. Schematic of events leading to the movement of the HRA. The hygroscopic expansion of the shell in vertical direction is selectively restricted on the vertical centreline, thereby forcing the edges to become longer than the centre. This causes the top and bottom edge to rotate with respect to one another, twisting the geometry. The constraints and the deformation they cause are also depicted in figure 2 .

still allowed in the $x y$-plane. See figure 2(a) for a schematic of the constraints applied to the design.

These selective restrictions impose a gradient on the amount of vertical elongation that is possible, being zero at the shell centre and increasing towards the shell edges. While this edge elongation causes a snap-through buckling with the petals of a lily [9], here it forces the shell to twist into a helicoid shape due to the right angle constraint. It thereby rotates the top and bottom edges with respect to each other, while staying parallel to the $x y$-plane (see figure 2(b)).

\subsection{Analytical model of geometrical parameters}

In the deformed state, the surface will take on a shape resembling a helicoid. With a known hygroscopic expansion, the theoretical achievable angle can be determined based on the main geometrical parameters $r$ (radius, measured from shell centreline to shell edge) and $h$ (height of shell, measured across its centreline) by looking at the length of the edge $L_{\mathrm{e}}$ of the surface.

In figure 3 , a helical curve is projected onto a cylindrical surface. This surface is then cut with this helical line and rolled out flat, so that a triangular shape is formed [24]. The length of this helix is defined by the height of the cylinder and the amount of circumferences the helix completes, using Pythagorean theorem.

$$
L_{i}=\sqrt{h^{2}+C_{i}^{2}}=\sqrt{h^{2}+(2 \pi i)^{2} \cdot r^{2}},
$$

where $h$ is the height of the cylinder, $C_{i}=2 \pi i \cdot r$ is the travelled distance in terms of a fraction $i$ of the circumference, $r$ is the radius of the base circle and $L_{i}$ is the length of the helix.

The movement of the presented design is driven by edge elongation and has rotation as the dependent variable. This elongation is caused by the hygroscopic strain of the material in vertical direction. Since the original geometry is a rectangular shell with vertical edges of equal length, the new length of the outer edge $L_{\mathrm{e}}$ after deformation is

$$
L_{\mathrm{e}}=h \cdot\left(1+S_{\mathrm{h}}\right) \text {, }
$$

where $S_{\mathrm{h}}$ is the engineering strain caused by hygroscopic swelling. 


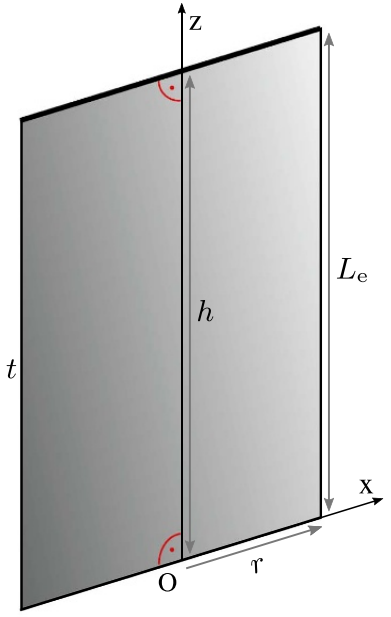

(a) Schematic of the HRA in undeformed state.

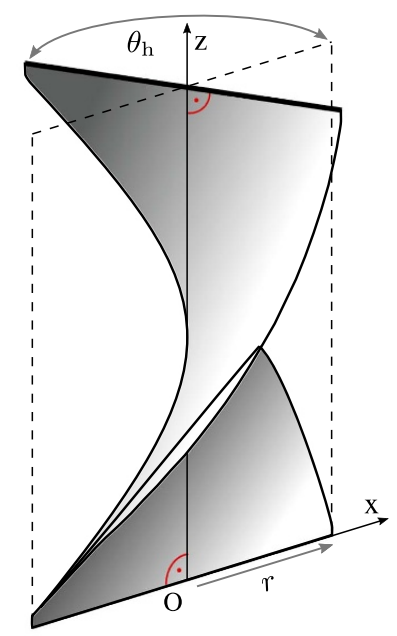

(b) Schematic of the HRA in deformed state, after being exposed to water
Figure 2. Schematic of the HRA, before (a) and after (b) being exposed to water. Radius $r$, height $h$ and thickness $t$ are the main geometric parameters that are being investigated. $\theta$ is the resulting angle. The movement is based on the differential elongation of the vertical edge $L_{\mathrm{e}}$ with respect to the length of the vertical centre $h$. The top and bottom horizontal edges are rigid and are forced to maintain a right angle with the vertical centreline.

Substituting equation (2) into equation (1) and rearranging terms to obtain the relation for an arbitrary rotation yields

$$
\begin{gathered}
h \cdot\left(1+S_{\mathrm{h}}\right)=\sqrt{h^{2}+(2 \pi i)^{2} \cdot r^{2}}, \\
2 \pi i=\sqrt{\frac{\left(h \cdot\left(1+S_{\mathrm{h}}\right)\right)^{2}-h^{2}}{r^{2}}} .
\end{gathered}
$$

This result can then be generalised to provide the angular rotation in degrees for each set of $S_{\mathrm{h}}, h$ and $r$ :

$$
\theta_{\mathrm{h}}=\frac{180}{\pi} \sqrt{\left(S_{\mathrm{h}}^{2}+2 S_{\mathrm{h}}\right) \frac{h^{2}}{r^{2}}} .
$$

This analytical model shows that the rotation due to hygroscopic swelling $\theta_{\mathrm{h}}$ is proportional to the height $h$ and inversely proportional to the radius $r$ of the shell, while being independent of its thickness $t$. The extent to which these relations capture the behaviour of an HRA is subject of investigation in the following sections.

\subsection{Numerical simulation}

To investigate the influence of the three main parameters on the performance of the HRA, numerical simulations are performed. The shell is modelled as a NURBS-surface using an isogeometric analysis (IGA) framework [25]. The KirchoffLove plate theory is used as constitutive relation and a linear orthotropic material law is applied. Furthermore, a quasi-static behaviour is assumed, meaning that dynamic effects are neglected. Thus the analysis consists of a series of static analyses

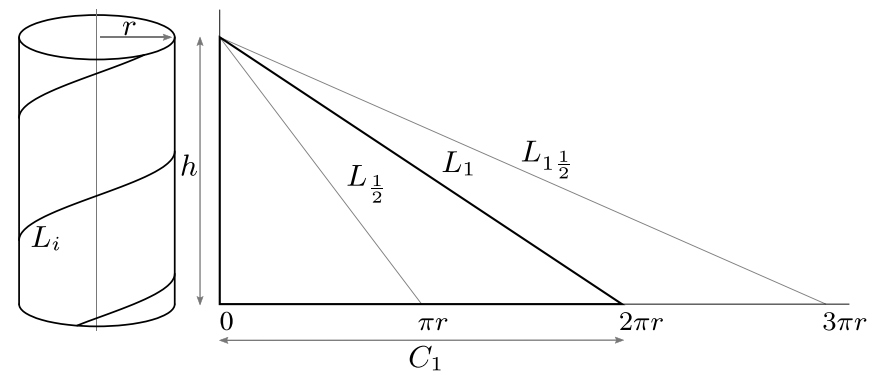

Figure 3. Schematic of dimensional properties of a rolled-out helix. The length $L_{i}$ of the helix is defined by the height $h$ of the cylinder that it envelops and the amount of circumferences $C_{i}=2 \pi \mathrm{i} \cdot \mathrm{r}$ it completes.

corresponding to the levels of applied strain. The non-linearity of the response is solved using a standard Newton-Rhapson algorithm. Only one half of the surface is simulated, since symmetry along the vertical axis is assumed.

Radius $r$ and height $h$ are discretized to 10 control points in u-direction (horizontal) and 100 in v-direction (vertical). To realize the constraints of the design, the control points along the bottom edge are fixed to restrict translation in all directions, realizing a clamping constraint. The control points on the top edge are constrained in $z$-direction only, allowing for movement in the $x y$-plane but maintaining the right angle between the central axis and top edge. To further simulate a clamping condition, the control points of both the top and bottom edges are rigidly connected to their vertical neighbours and these connections are forced to maintain a right angle with the $x y$-plane.

To enable symmetry, the nodes along the centreline are constraint in all directions of translation. Their rotations are unconstrained in order to allow the surface to twist. The top and bottom control points of the vertical centreline are defined as clamping points and can be used to measure or impose torques and rotations about the $z$-axis. See figure 4(b) for an overview of the constraints imposed on the model.

A main geometry is chosen, with dimensions $30 \mathrm{~mm}$ by $70 \mathrm{~mm}$ by $0.62 \mathrm{~mm}(r \cdot h \cdot t)$. From this geometry, radius, height and thickness are independently varied in positive and negative direction to generate a variety of geometries. For each of these geometries, the hygroscopic engineering strain $S_{\mathrm{h}}$ is imposed on the shell in the (initially vertical) $v$-direction, by stepwise increasing the strain on element-level. In essence, the imposed strain value changes the neutral length of the elements but does not directly impose a given length to the elements in the deformed state. The stresses that result from the change in neutral length drive the deformation. As the geometry deforms, the resulting angle of the HRA can be determined. An initial disturbance and a small initial twist are implemented to help the simulation overcome buckling, and to force the deformation to be anticlockwise to its undeformed state (see figure 2).

To compare the angles resulting from the analytical model with those resulting from the numerical simulations for each parameter of interest, a Root Mean Square Error (RMSE) is used. It is defined as 


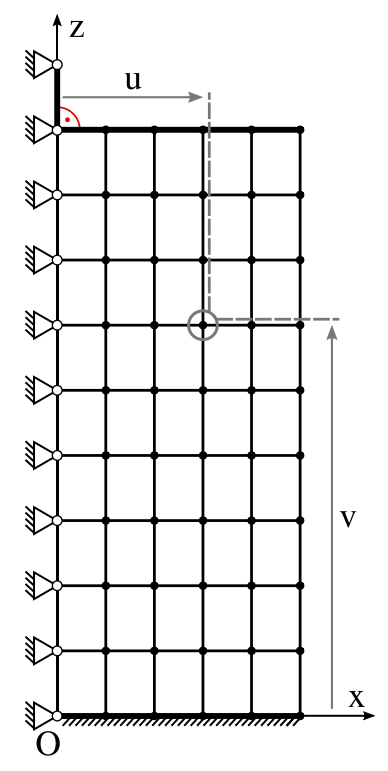

(a) Undeformed model, 2D view. The parametric coordinates $\mathrm{u}$ and $\mathrm{v}$ of an arbitrary node are shown.

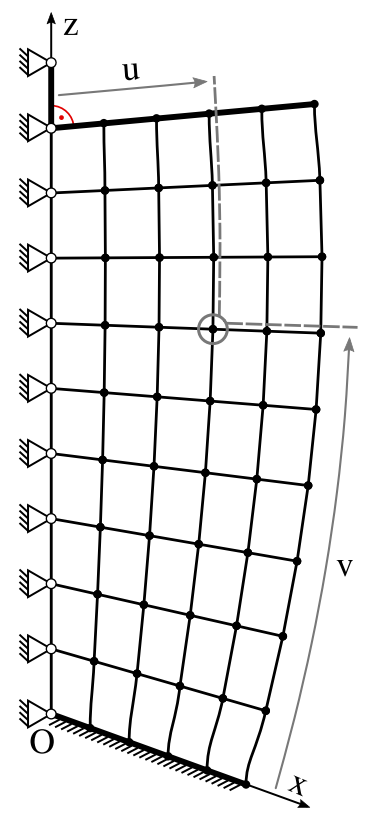

(b) Deformed model, 3D view. The parametric coordinates $u$ and $\mathrm{v}$ of the node move with the shell.

Figure 4. Schematic of the geometry as implemented in the numerical model. A surface is parameterized by defining nodes in $u$-direction and $v$-direction. By definition, these directions are tangent to each location of the surface. The implemented translational constraint along the vertical centreline and the clamping constraints along the horizontal top and bottom edges are also shown.

Table 1. Dimensions of the geometries that were subjected to torque-simulations. Versions denoted in capitals (A-E) were also produced and tested as prototypes.

\begin{tabular}{lccc}
\hline Version & $\begin{array}{c}\text { Radius } r \\
(\mathrm{~mm})\end{array}$ & $\begin{array}{c}\text { Height } h \\
(\mathrm{~mm})\end{array}$ & $\begin{array}{c}\text { Thickness } t \\
(\mathrm{~mm})\end{array}$ \\
\hline $\mathrm{A}$ & 30 & 70 & 0.62 \\
$\mathrm{~B}$ & 30 & 40 & 0.62 \\
$\mathrm{C}$ & 30 & 100 & 0.62 \\
$\mathrm{D}$ & 10 & 70 & 0.62 \\
$\mathrm{E}$ & 50 & 70 & 0.62 \\
$\mathrm{~F}$ & 30 & 70 & 0.32 \\
$\mathrm{G}$ & 30 & 70 & 1.22 \\
\hline
\end{tabular}

$$
\mathrm{RMSE}=\sqrt{\frac{1}{n} \sum_{i=1}^{n}\left(\theta_{\mathrm{ha}, i}-\theta_{\mathrm{hs}, i}\right)^{2}}
$$

where $n$ is the amount of solved geometries for each parameter of interest. Angles $\theta_{\mathrm{ha}, i}$ and $\theta_{\mathrm{hs}, i}$ are the analytical and numerical resulting angles of geometry $i$, respectively.

To investigate torque, seven deformed geometries are chosen (table 1): the main geometry, and for each design parameter a value near the beginning and end of the simulated range. In the table, the geometries that were also used for the prototypes are denoted in capitals. A rotational displacement is gradually imposed on the upper clamping point of the surface,

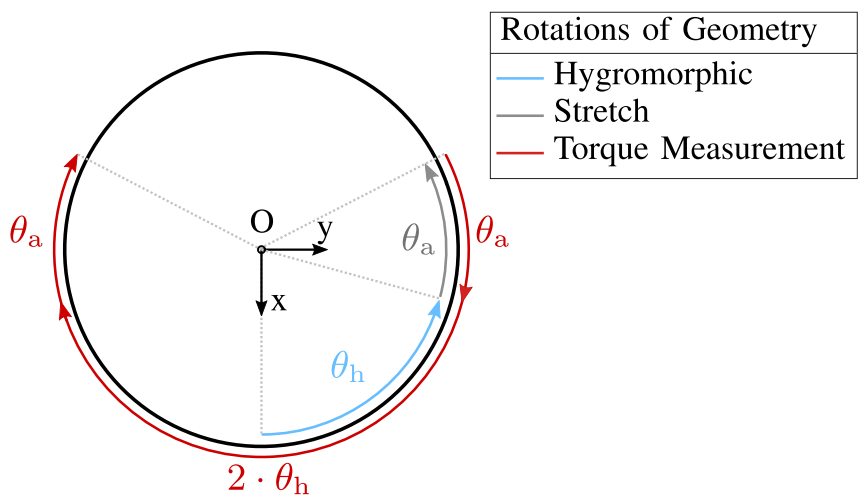

Figure 5. Schematic showing the different rotations subjected to the geometries, depicted from above. The hygromorphic rotation $\left(\theta_{\mathrm{h}}\right)$ is caused by exposure to water. The stretching rotation $\left(\theta_{\mathrm{a}}\right)$ and the torque measurement rotation $\left(2 \theta_{\mathrm{a}}+2 \theta_{\mathrm{h}}\right)$ are caused by applying a rotation on the top clamping point.

while measuring the torque exerted on the bottom clamping point about the z-axis. In this part of the analysis, the NewtonRhapson solver is displacement-driven. This overcomes possible difficulties to go by limit-points of the solution curve caused by, e.g. multi-stability of the system.

This rotation consists of two steps. Before starting to measure the torque on the bottom clamping point, an additional anticlockwise rotation $\theta_{\mathrm{a}}$ of $30^{\circ}$ is imposed, stretching the geometry beyond its deformed state. Second, a clockwise rotation is imposed while measuring the torque. This final clockwise rotation consists of twice the resulting angle $\theta_{\mathrm{h}}$ and twice the additional angle $\theta_{\mathrm{a}}$, stretching the geometry again. These steps are taken to determine the behaviour beyond the hygromorphic deformation. Figure 5 shows the complete sequence of rotation, including the initial resulting angle $\theta_{\mathrm{h}}$.

\subsection{Prototype realisation}

In order to validate the simulations, five different geometries were built and tested. These geometries were chosen to correspond with the choices made for the simulated geometries. The central geometry A was built, as well as geometries towards the ends of the simulated spectrum of height (geometries $\mathrm{B}$ and $\mathrm{C}$ ) and radius (geometries D and E), in both positive and negative direction. The thickness has not been varied in the physical tests, due to the lack of availability of veneer slices with the same properties but different thickness. See table 1 for an overview of the dimensions of the prototypes.

Because of its orthogonal properties, most notably the direction-dependant expansion [23], wood was chosen as hygro-responsive material. Performance of thin slices of wood (veneer) has proven to be sufficient for actuator applications [19]. When exposed to water, these veneer slices elongate perpendicular to their fibre direction only; they maintain their length parallel to the fibre.

The veneer slices were glued in 3D printed PLA clamps (Prusa Mk3s) with their fibres oriented horizontally. These clamps keep a perpendicular angle between the vertical centreline and the top and bottom edges of the shell. The 
Table 2. Overview of the dry and wet material properties used in the numerical simulations.

\begin{tabular}{llccc}
\hline & \multicolumn{2}{c}{ Young's modulus } & Shear modulus & Poisson's \\
& $E_{\mathrm{L}}(\mathrm{MPa})$ & $E_{\mathrm{T}}(\mathrm{MPa})$ & $G_{\mathrm{LT}}(\mathrm{MPa})$ & $\begin{array}{c}\text { Strain } \\
E_{\mathrm{T}}(-)\end{array}$ \\
\hline Dry & 5619.5 & 207.2 & 356.9 & 0.048 \\
Wet & 4369.2 & 174.2 & 277.4 & 0.048 \\
\hline
\end{tabular}

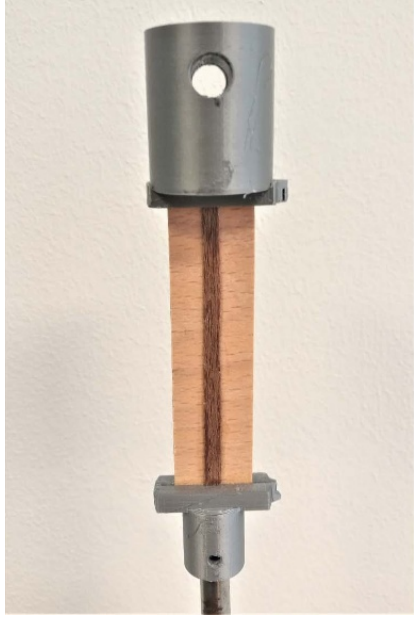

(a) A specimen of prototype D in dry, undeformed state.

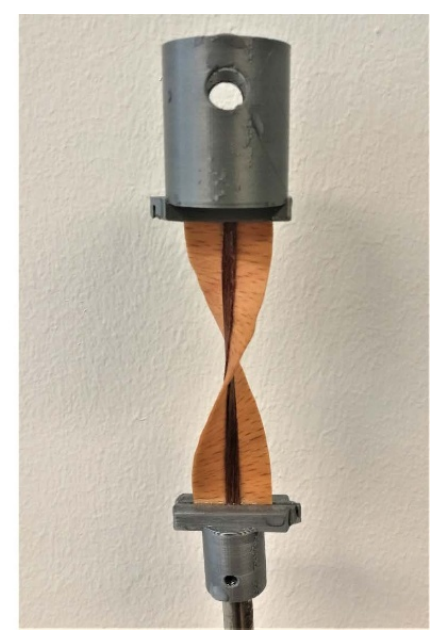

(b) A specimen of prototype D in wet, deformed state.
Figure 6. Dry and wet state of prototype $\mathrm{D}$, with a radius of $10 \mathrm{~mm}$ and a height of $70 \mathrm{~mm}$. The thin slice of darker wood serves as the centreline constraint and is glued on the veneer at both sides. The grey $3 \mathrm{D}$ printed parts serve both as constraint of top and bottom edge, and as connection interface between prototype and torsion machine.

necessary constraint along the vertical centreline was realized by taking two narrow rods of wood with the fibre in the vertical direction and gluing them to the middle of the veneer on either side. This bond of wood on wood proved to maintain its integrity in most cases, even after being exposed to water with the resulting large deformations. An example of a produced prototype is shown in figure 6.

\subsection{Prototype material properties}

To investigate the behaviour of the HRA experimentally, and to validate the analytical model and the numerical analysis, prototypes were made. Beech veneer was chosen as the hygroactive material for the HRA prototypes. In preliminary tests, it showed to have a sufficient elongation while maintaining its structural integrity during large deformations.

Some material properties needed to be determined for implementation in the orthotropic material model, before it could be used in the numerical simulations. Hygroscopic expansion was measured by soaking five slices of veneer in water and measuring their dimensions before and after soaking. This resulted in an average elongation of $8.4 \%$ perpendicular to the fibre direction. No elongation parallel to the fibre direction occurred.

The Young's moduli were determined by performing a three-point bending test on five slices of veneer in both dry and wet state, resulting in a Young's modulus perpendicular to the fibre $\left(E_{\mathrm{T}}\right)$ of $174.2 \mathrm{MPa}$ and a Young's modulus parallel to the fibre $\left(E_{\mathrm{L}}\right)$ of $4369.2 \mathrm{MPa}$.

These values are lower than those found in literature, although values in literature vary substantially [26-28]. This is potentially due to the fact that in this case, the wood is completely saturated, whereas values in literature are based on a lower water content [27]. Furthermore, the wood used could be a different species of beech with a different density. Young's moduli of wood are known to decrease with increasing water content and vary with different species and densities [23].

The shear modulus $G_{\mathrm{LT}}$ and Poisson's ratio $\nu_{\mathrm{LT}}$ are needed. The shear modulus was determined by calculating the ratio between mean values of $E_{\mathrm{L}}$ and $G_{\mathrm{LT}}$ from literature [26] and applying this ratio to the experimentally determined $E_{\mathrm{L}}$ of the veneer, resulting in a value of 781.3 MPa. Since the Poisson's ratio does not vary substantially with varying water content, it was determined from literature [26], resulting in a value of 0.048 . An overview of the material properties is given in table 2 .

\subsection{Test protocol}

Each of the five prototype variations was fabricated five times, resulting in a total of 25 specimens. Each specimen was first soaked in water until no further rotation was noticed. It was then photographed from above. The resulting angle $\theta_{\mathrm{h}}$ was determined using the image analysis software ImageJ [29].

Next, the specimen was placed in a torsional test bench (Zwick Z005 with HBM T20WN 0.5 Nm torque meter). A rotation was gradually imposed, while measuring the torque $T$ on the bottom clamping point around the vertical axis. The same approach was taken as with the simulations, with additional rotations beyond the resulting angle $\theta_{\mathrm{h}}$ of the deformed geometry (see figure 5). In this case however, a smaller $\theta_{\mathrm{a}}$ ranging from $5^{\circ}$ to $2^{\circ}$ was applied, so that the prototypes would not break before test completion.

From the angle measurements, boxplots were created to be able to compare the experimental resulting angles to the angles derived from the analytical model and the numerical simulations. For each of the prototypes A to E, the torque-rotation graphs of the successful specimen were interpolated to obtain the average torque-rotation graph for that specimen. These graphs could then be compared to the results of the torque simulations.

\section{Results}

\subsection{Hygromorphic rotation}

Figure 7 shows the angle resulting from the simulations for increasing height (orange dots, figure a) and radius (blue dots, 
figure b) respectively. Additionally, the grey lines show the resulting angle according to the analytical model, calculated using equation 5 .

The numerically simulated angles from geometries with varying height and radius follow the same path as the analytically calculated angles, albeit with a difference. The Root Mean Square Error (RMSE) between the analytically and numerically calculated angles when varying the height is $5.4^{\circ}$. When varying the radius, the RMSE is $8.1^{\circ}$.

The influence of the thickness $t$ on the resulting angle is depicted in figure 8 . The numerically calculated angle fluctuates around the constant angle predicted by the analytical model, with an RMSE of $4.7^{\circ}$.

The development of the vertical engineering strain at each horizontal location of the geometries $\mathrm{A}$ to $\mathrm{E}$ is depicted in figure 9, normalised as a percentage of the radius. Although the initial vertical strain imposed on the shell is 0.084 along its entire radius, the resulting vertical strain calculated from the actual elongation is not uniform along the radius. As imposed by the constraints, it is zero at the shell centreline. It increases towards the shell edge and overshoots the imposed strain. The amount of overshoot increases when radius decreases, being $50 \%$ in the case of geometry D.

The graphs predicted by the numerical simulations show discontinuities. Figures 10(a) and (b) show the geometries highlighted in figure 7(b), before and after a discontinuity with a drop in resulting angle. These geometries differ in radius by only $0.1 \mathrm{~mm}$, while having a difference in resulting angle of $15.51^{\circ}$.

Taking a closer look at the deformed geometries at these discontinuities of the numerical simulation graphs, different buckling modes and edge wrinkles can be seen. A simplified representation of the wrinkling of the edge per segment of the resulting angle graph obtained from the numerical simulation is shown in the insets in figures 7 and 8.

This edge-wrinkling is present to varying extent at all solved geometries. As seen in figure 7(b), the shell edge maintains a similar shape per segment when increasing radius. At small radii a few wrinkles with a small amplitude are present, growing in size and numbers at segments with larger radii. Varying height (figure 7(a)) shows a different behaviour. Here, the end of a segment shows multiple larger wrinkles, which get smoothed out when jumping to a next segment with a higher resulting angle. When the height increases further, wrinkles form again, until the next jump in resulting angle.

The boxplots in figure 7 show the angular deformation of the tested prototypes. They show the same behaviour when radius or height varies, but their resulting angle is on average $20 \%$ lower than that of the numerical simulations. An example of a hygroscopically deforming prototype is displayed in movie $\mathrm{S} 1$ (is available online at stacks.iop.org/SMS/30/025008/mmedia), showing a timelapse of a specimen of prototype $\mathrm{D}$ being exposed to water.

The prototypes also show similar buckling modes and wrinkling behaviour as the simulations. Within the same prototype there is a spread in resulting angle. This is most notable when looking at various specimen of prototype $\mathrm{E}$, depicted in

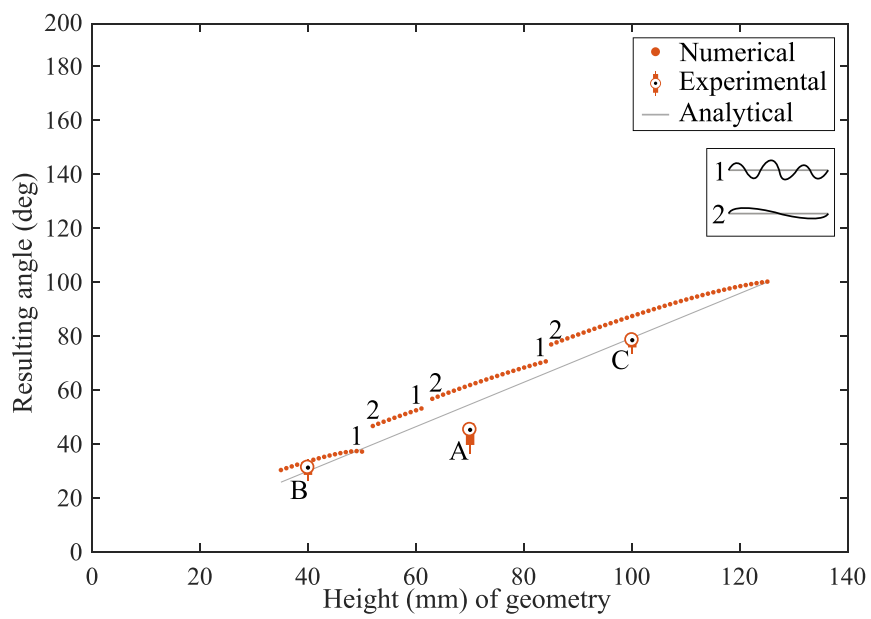

(a) Resulting angle of geometries with constant radius and varying height.

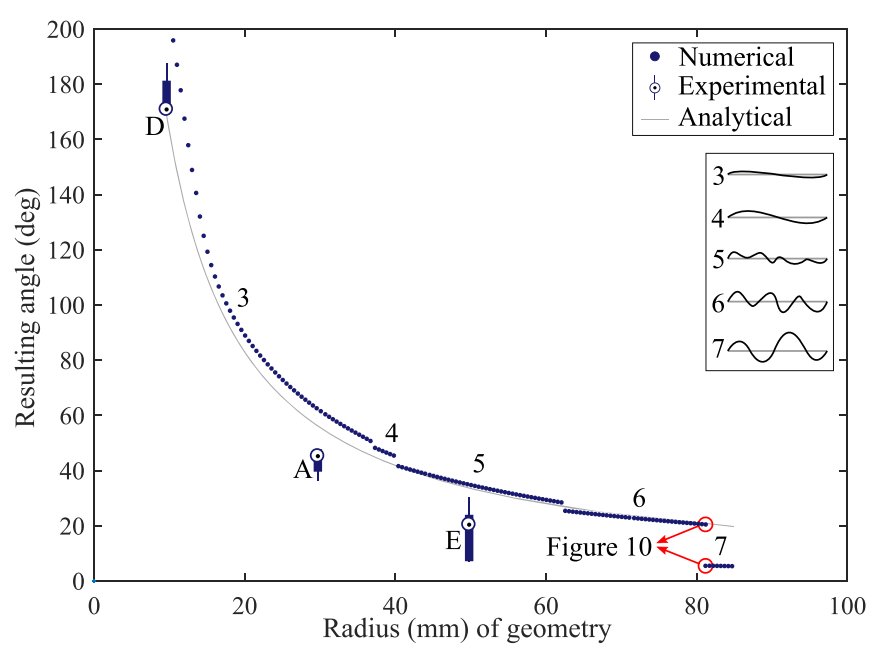

(b) Resulting angle of geometries with constant height and varying radius.

Figure 7. Resulting angle of geometries with constant radius and varying height (figure a), and geometries with constant height and varying radius (figure b). Results of the simulation are depicted using dots. The grey lines show the angle from the theoretical calculation (equation 5). The results of the tested geometries are depicted with boxplots, with their labels corresponding to the prototype versions in table 1 . The insets show a $2 \mathrm{D}$-representation of the wrinkles of the edge at the numbered segments of the graphs.

figures $10(\mathrm{c})$ and (d). Its resulting angle ranges from $7.1^{\circ}$ to $30.5^{\circ}$.

\subsection{Torque upon applied rotation}

The torque-rotation graphs of the five main geometries have been determined both numerically and experimentally. The left column of figure 11 shows the hygromorphically deformed geometry that is used as starting point for the torque simulations, together with the undeformed main geometry A (red outline). The middle column shows the numerical results (orange line) and the interpolated result of the tests (blue line) for each of the five geometries A to E. Additionally, the grey lines show the simulated torque during a rotation of a dry and undeformed shell. 


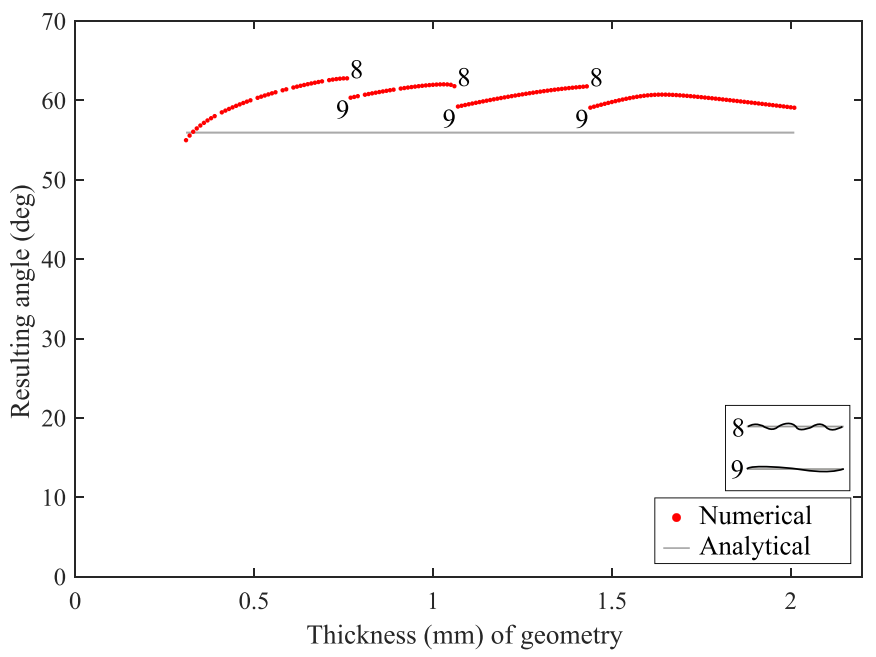

Figure 8. Resulting angle of geometries with constant radius and height, and varying thickness.

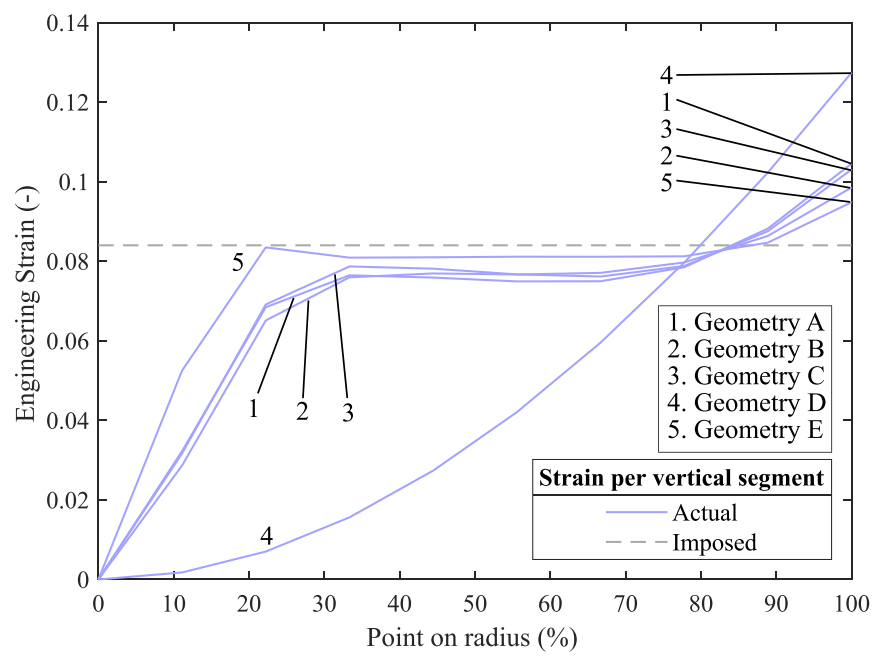

Figure 9. Engineering strain per vertical segment of the shell, for each of the five simulated geometries A-E. The grey dashed line represents the strain imposed on the shell in v-direction. At $100 \%$ of the radius, the strains of the geometries overshoot the imposed strain. Note that the radii of the five geometries are normalized.

The right column shows how each of the interpolated lines originates from the tested specimen. The grey lines show the different specimen that were tested per geometry. The torques of the tested geometries have been scaled by 0.5 , since the prototypes consisted of two shells and the simulations only of one due to the symmetry across the centreline. Note that not all tests succeeded; some specimen failed during hygroscopic deformation, some failed halfway through the rotation sequence. Each tested prototype has at least three successfully tested specimen.

During rotation, the torques of the wet geometry are close to zero. As soon as the resulting angle is reached, however, torque increases. The dry rotation shows this increase immediately when being rotated, without a region of zero torque.

No real trend is observed when looking at the torque graphs of different radii or heights, other than the transition region

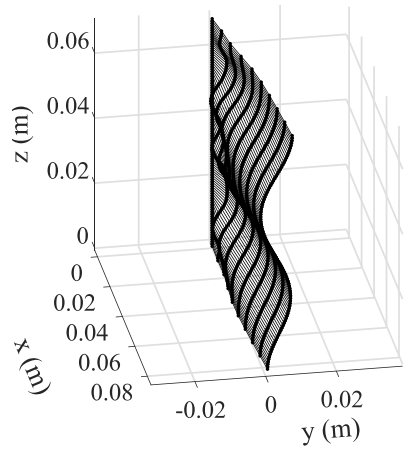

(a) Deformed geometry with a radius of $81.35 \mathrm{~mm}$. Note the wrinkles of the shell edge, resulting in a rotation of 5.65 degrees.

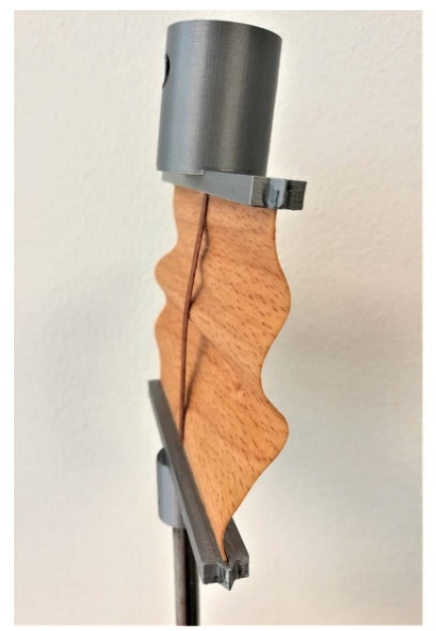

(c) A specimen of prototype $\mathrm{E}$ in wet state, with a rotation of 7.1 degrees. Debonding between the shell and the centreline constraint is

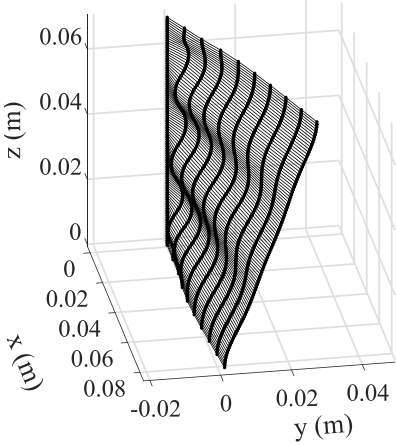

(b) Deformed geometry with a radius of $81.25 \mathrm{~mm}$. Note the smoother edge, resulting in a rotation of 21.16 degrees.

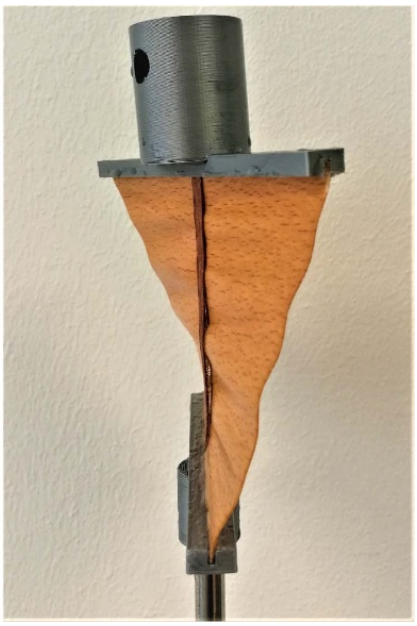

(d) A specimen of prototype $\mathrm{E}$ in wet state, with a rotation of 30.5 degrees. present.

Figure 10. Comparison of buckling modes of deformed geometries. Figures (a) and (b) show an example of a simulated geometry with a slightly different radius just before (a) and after (b) a discontinuity in resulting angle. The specific moment of this example is depicted in figure 7 with red circles. Figures (c) and (d) both show a wet specimen of prototype $\mathrm{E}$, with a radius of $50 \mathrm{~mm}$ and a height of $70 \mathrm{~mm}$.

from positive to negative torque being larger with a larger resulting angle. The magnitude of the transition region of geometry $\mathrm{E}$ is highest, followed by geometry $\mathrm{C}$.

The prototypes show similar behaviour. Their graphs show a region of low torque, too. The torque in their region has smaller fluctuations but generally has a positive offset, whereas the torque in the transition region of the simulated geometries fluctuates around zero. The magnitude of the transition region fluctuates around $0.007 \mathrm{~N} \cdot \mathrm{m}$ for prototypes $\mathrm{A}, \mathrm{B}$ and $\mathrm{D}$, around $0.015 \mathrm{~N} \cdot \mathrm{m}$ for prototype $\mathrm{C}$, and around $0.02 \mathrm{~N} \cdot \mathrm{m}$ for prototype E.

As shown in figure 12, the rotation-torque graphs of geometries with a varying thickness following from the simulations show similar behaviour as those of the rotation-torque graphs of geometries with varying radius and height. Within 

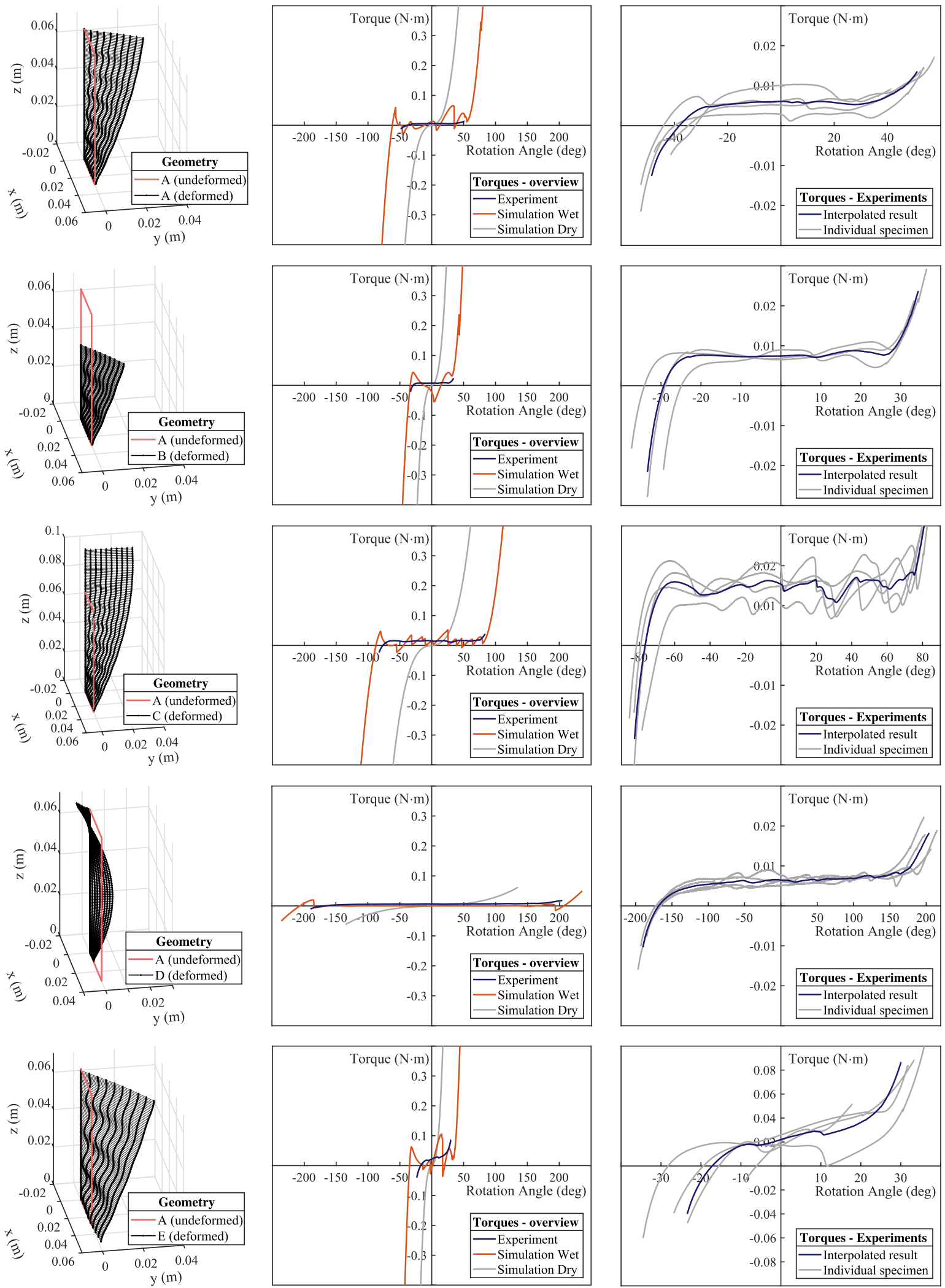

Figure 11. Deformed geometries and torque-graphs of the five simulated and tested geometries. The left column displays the hygroscopicly deformed geometries before being rotated back, together with the outline of the undeformed main geometry A in red. The middle column displays the torques of simulated geometries (orange lines) and the interpolated result of the tested prototypes (blue lines). Additionally, the simulated torque of a dry and undeformed geometries is shown in grey. The right column shows how the interpolated line for each prototype is determined from the tested specimen. Note that the scale of the $y$-axis is different from the one in the middle column. 


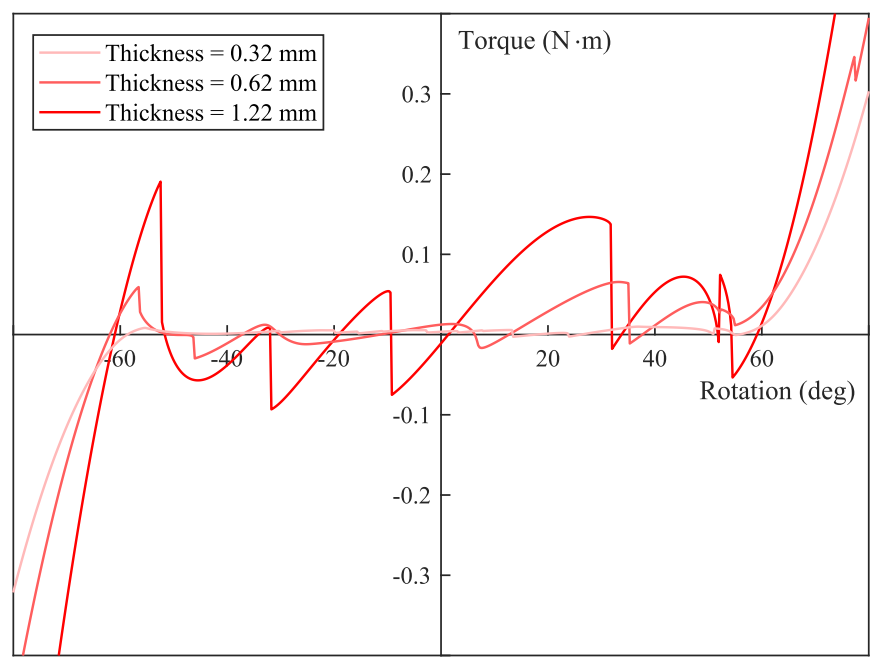

Figure 12. Torque during simulated back-rotation of three instances of geometry A, each with a different thickness.

the hygromorphically deformed angular range, torque fluctuates around zero. Rotating the geometry beyond this range increases the torque. The height of the fluctuations does vary however. The peak-to-peak distance increases as the thickness of the shell increases.

\section{Discussion}

\subsection{Influence of geometric parameters on resulting angle}

Looking at figures 7(a) and (b), height and radius prove to be valid parameters to design for a certain rotation of an HRA. Depending on the specific application and corresponding size, one of the two can be chosen, after which the other follows from the desired angular output.

The numerical simulations confirm the relation between $h$, $r$ and $\theta_{\mathrm{h}}$ from the analytical model of equation 5. The resulting angle $\theta_{\mathrm{h}}$ increases proportionally as height $h$ increases, and decreases as radius $r$ increases. Unlike suggested by the analytical model, figure 8 shows that the resulting angle is not entirely independent of the thickness of the shell. Due to the increasing and decreasing number of wrinkles of the shell, the resulting angle fluctuates around the analytical value when thickness is increased. This fluctuation is minimal however.

The difference between the resulting angles found in the analytical model and the numerical simulations can be explained by looking at the development of the strains in figure 9. The actual vertical engineering strain overshoots the imposed engineering strain $S_{\mathrm{h}}$ at the shell edge. This means that the length of the outer edge of the shell $L_{\mathrm{e}}$ will be longer than calculated in equation 2, resulting in a larger rotation.

Another difference between the analytical model and the numerical simulations, are the discontinuities in the graphs of the simulated angles. Taking the deformed geometries in figure 10 as an example, shown in figure 7 with red circles, a reason for these discontinuities can be seen. Although their radii differs only by $0.1 \mathrm{~mm}$, the geometry in figure 10 (a) buckles extensively, resulting in wrinkles along its edge. The part of the elongation that is used to create those wrinkles is not converted in a rotation. The edge of the geometry in figure 10(b), however, is almost smooth and therefore a larger resulting angle is achieved.

Each line segment of the numerical calculations in figures 7 and 8 is thought to correspond to a certain buckling mode of the shell. Multiple parallel lines may exist, from which only segments are found when the numerical simulation jumps from one to the other. Multiple modes can exist in the same geometry, meaning that the same geometry can have different stable deformed instances, each of them with a different resulting angle. This behaviour resembles that of other multistable compliant shell mechanisms [30-33] and is shown in movie S2. What exactly causes this multi-stable behaviour and how it can be designed to fully exploit its benefits, remains subject for future research.

The observed buckling modes and multi-stability are also present within multiple specimen of the same prototype. Some specimen display a small angle with large wrinkles on the edge, but can be manually rotated to their maximum angle with smooth edge and maintain this position. Figures $10(\mathrm{c})$ and (d) show an example of this phenomenon. The specimen of prototype $\mathrm{E}$ in figure $10(\mathrm{c})$ displays more and larger wrinkles, converting less elongation in a rotation than the specimen of prototype $\mathrm{E}$ in figure $10(\mathrm{~d})$.

\subsection{Influence of geometric parameters on torque}

Once deformed, the HRA behaves like a zero-moment element, essentially straightening and elongating the crossover region from positive to negative torque. In its wet and deformed state, the geometry can be rotated back with a torque close to zero, until the negative hygromorphic angle is reached.

Radius and height do not seem to have an influence on the magnitude of the torque, besides the fact that the zero-moment region is larger with a smaller radius or larger height. The thickness of the shell does have an influence on the peak-topeak value of the oscillations in the zero-moment region of the rotation and on the slope of the rotation-torque graph outside of the zero-moment region. Both increase as thickness increases, suggesting that thickness can be used to tweak the torque-output during rotation. Further research is needed to quantify the influence of thickness on the torque.

The fluctuations of the torque in the zero-moment region of both the simulated and tested geometry are caused by wrinkles being formed or being stretched flat. This is displayed in more detail in movie S3, showing the back-rotation of a specimen of geometry $\mathrm{C}$ together with its torque-graph. Initially, a wrinkle will resist rotation, causing an increase in torque. Beyond a certain threshold, the shell rapidly jumps to another mode, releasing the build up stress. This manifests itself in the torque graphs as fluctuations around a value close to zero.

The positive offset of the torque graphs of the prototypes is supposedly due to the visco-elastic behaviour of the wood. The angle-torque graphs depend on the direction of the rotation. The graphs in the right column of figure 11 only show one direction; if the geometry would have been rotated back to the start position of the torque-test, a loop would form. 
More research is needed to further investigate the visco-elastic aspect of wood as a material for the HRA.

\subsection{Comparing numerical simulation and prototype experiments}

The observed differences between numerical simulations and experiments are small. The biggest difference lies within the zero-moment region of the torque graphs, which has bigger fluctuations in the simulations. The geometry is modelled as a perfect uniform shell, whereas the veneer used in the prototypes has imperfections. A perfect shell buckles more abruptly than an imperfect shell. This could explain the differences observed in the torque-graphs. Further investigation may improve simulation accuracy.

The lower resulting angles of the tested geometries can be explained by looking at the differences between the simulated model and the constructed prototypes. The most notable difference is the implementation of the constraint. The added thin slices of wood at each side of the shell stop the middle from elongating, but they also add extra rotational stiffness. This additional stiffness will resist angular deformation and therefore the deforming geometry reaches a smaller resulting angle. Likewise, the bonding between the slices of wood and the shell sometimes fails as a result of large buckling, as can be seen in figure 10(c). This also causes a lower resulting angle.

The choice of veneer as a material for the HRA has proven to be advantageous for the creation of an initial prototype. It is easily available and low-tech. However, some challenges arise from its inhomogeneity and unpredictability. Wood is known to have imperfections, which in this case causes a spread in behaviour between specimen and sometimes specimen fail before test completion. After two to five cycles, most prototypes were broken. Furthermore, the shell is not perfectly symmetrical across its centreline because of these imperfections, causing differences in the shape of the edge wrinkles on either side. The general movement remains symmetrical however, due to the rigid clamps on the top and bottom edges. Investigating different hygroscopic materials that remain intact during repeated movement cycli will greatly improve prototype performance.

Additionally, the form-freedom of veneer is limited to flat or developable shells. For some applications it would be beneficial to develop a more controllable material that can be shaped to a spatially curved shell, while maintaining its hygroscopic directional properties.

\subsection{Potential applications}

The geometries treated in this paper can be of use in a number of applications. According to the analytical model, equal scaling of height $h$ and radius $r$ yields the same resulting angle $\theta_{\mathrm{h}}$. Scaling will be limited by material availability however, as the circular cross-section of trees limits the size of veneer with parallel fibres that can be produced. To design an HRA for larger applications, a different hygro-active material might be needed.
Similar to already existing systems [19], multiple HRA's could be placed on a building façade, regulating the airflow through the building in response to the environmental humidity. Due to the cylindrical movement space, they can be placed inside a pipe and will therefore stick out less than existing meteoro-sensitive façades.

Developing the placement inside pipes even further, the HRA could also be used to regulate waterflow in for instance irrigation systems. It can be designed to open a container of water during a period of drought, closing the container again when a certain volume of water has passed. A precise control of the rotation based on the influx of water in the material will be needed.

Alternatively, a different look on the HRA might provide another application. In zero-moment systems, also referred to as neutrally stable systems, pretension is needed to perform the zero-moment behaviour. By fixing both ends of the shell, the HRA provides a way of adding or subtracting pretension, thereby activating the system to enable its functionality.

Finally, the concept of a rotational motion due to selective restriction of an expansion does not limit itself to hygroscopic swelling only. Passive actuators could be designed to take advantage of other environmental gradients such as temperature, making the concept accessible for more high-tech applications and materials.

\section{Conclusion}

A Hygromorphic Rotational Actuator has been designed by selectively restricting the direction-dependent hygroscopic elongation of a compliant shell. The HRA is capable of deforming its own geometry, and therefore matches the observed behaviour in the plant world and that of other hygromorphic actuators. The simplicity of the HRA is its greatest asset; it operates without the need of additional sensors, motors and control units, and it does so with a minimum amount of parts.

The presented design adds to the possibilities of passive hygromorphic actuators and can be used as a building block in larger compliant systems. Its range of motion can be tweaked by selecting the radius and height of the shell. The shell's thickness does not have a notable influence on its kinematic behaviour. It does seem to influence the magnitude of the torque. This effect should be investigated in more detail.

The multi-stable and zero-moment behaviour are interesting aspects of the HRA that can further expand its usage. Being able to precisely control these aspects remains subject for future research,

\section{Acknowledgments}

The authors would like to acknowledge NWO (P16-05: Shell Skeletons) for the financial support of this project. The authors would also like to thank the lab technicians of the Department of Precision and Microsystem Engineering of the TU Delft for providing help and advice in the design of the experimental setup. 


\section{ORCID iDs}

Sebastiaan Joosten (D) https://orcid.org/0000-0002-28677297

Giuseppe Radaelli (D) https://orcid.org/0000-0002-2935-3868

Heike Vallery (D) https://orcid.org/0000-0002-0305-398X

\section{References}

[1] Sakes A, Van Wiel M D, Henselmans P W, Van Leeuwen J L, Dodou D and Breedveld P 2016 Shooting mechanisms in nature: a systematic review PloS One 11 1-46

[2] Elbaum R, Zaltzman L, Burgert I and Fratzl P 2007 The role of wheat awns in the seed dispersal unit Science 316 884-6

[3] Joosten S, Radaelli G and Vallery H 2019 Compliant shell deformation in plants: a designer's toolbox Delft University of Technology (http://resolver.tudelft.nl/uuid:d04ae32273ad-4f27-bfb5-5cead96a8e65)

[4] Dumais J and Forterre Y 2011 Vegetable dynamics: the role of water in plant movements Annu. Rev. Fluid Mech. 44 453-78

[5] Charpentier V, Hannequart P, Adriaenssens S, Baverel O, Viglino E and Eisenman S 2017 Kinematic amplification strategies in plants and engineering Smart Mater. Struct. 26063002

[6] Elbaum R 2018 Structural principles in the design of hygroscopically moving plant cells Plant Biomechanics: From Structure to Function at Multiple Scales, eds A Geitmann and J Gril (Sainte-Anne-de-Bellevue, QC: Springer Int. Publishing) pp 235-45

[7] Reyssat E and Mahadevan L 2009 Hygromorphs: from pine cones to biomimetic bilayers J. Royal Soc. Interface 6 951-7

[8] Hayashi M, Gerry S P and Ellerby D J 2010 The seed dispersal catapult of Cardamine parviflora (Brassicaceae) is efficient but unreliable Am. J. Bot. 97 1595-601

[9] Liang H and Mahadevan L 2011 Growth, geometry and mechanics of a blooming lily Proc. Natl Acad. Sci. $1085516-21$

[10] Kobayashi H, Kresling B and Vincent J F 1998 The geometry of unfolding tree leaves Proc. R. Soc. B 265 147-54

[11] Speck T, Bold G, Masselter T, Poppinga S, Schmier S, Thielen $\mathrm{M}$ and Speck O 2018 Biomechanics and Functional Morphology of Plants-Inspiration for Biomimetic Materials and Structures Plant Biomechanics: From Structure to Function at Multiple Scales 1st ed, eds A Geitmann and J Gril (Cham: Springer Int. Publishing) ch. 4, pp 399-433

[12] Schleicher S, Lienhard J, Poppinga S, Speck T and Knippers J 2015 A methodology for transferring principles of plant movements to elastic systems in architecture CAD Comput. Aided Des. 60 105-17

[13] Poppinga S, Zollfrank C, Prucker O, Rühe J, Menges A, Cheng T and Speck T 2018 Toward a new generation of smart biomimetic actuators for architecture Adv. Mater. 30 1-10

[14] Burgert I and Fratzl P 2009 Actuation systems in Plants as prototypes for bioinspired devices Phil. Trans. R. Soc. A 367 1541-57
[15] Rüggeberg M and Burgert I 2015 Bio-Inspired wooden actuators for large scale applications PLoS One 10 1-16

[16] Zhang L, Chizhik S, Wen Y and Naumov P 2016 Directed motility of hygroresponsive biomimetic actuators $A d v$. Funct. Mater. 26 1040-53

[17] Al-Obaidi K M, Azzam Ismail M, Hussein $\mathrm{H}$ and Abdul Rahman A M 2017 Biomimetic building skins: an adaptive approach Renewable Sustainable Energy Rev. 79 1472-91

[18] Wood D M, Correa D, Krieg O D and Menges A 2016 Material computation-4D timber construction: towards building-scale hygroscopic actuated, self-constructing timber surfaces Int. J. Archit. Comput. 14 49-62

[19] Reichert S, Menges A and Correa D 2015 Meteorosensitive architecture: biomimetic building skins based on materially embedded and hygroscopically enabled responsiveness CAD Comput. Aided Des. 60 50-69

[20] Shin B, Ha J, Lee M, Park K, Park G H, Choi T H, Cho K J and Kim H Y 2018 Hygrobot: a self-locomotive ratcheted actuator powered by environmental humidity Sci. Robot. $31-9$

[21] Raviv D et al 2014 Active printed materials for complex self-evolving deformations Sci. Rep. 41-8

[22] Erb R M, Sander J S, Grisch R and Studart A R 2013 Self-shaping composites with programmable bioinspired microstructures Nat. Commun. 4 1-8

[23] Kretschmann D E 2010 Mechanical Properties of Wood Wood Handbook-Wood as an Engineering Material, centennial, ed R J Ross ch. 5 (Madison, WI: U.S. Dept. of Agriculture, Forest Service, Forest Products Laboratory) p 5-1-5-45 (available at: www.fpl.fs.fed.us)

[24] Steinhaus H 1983 Squirels, screws, candles, tunes and shadows Mathematical Snapshots 3rd edn (Mineola, New York: Dover Publications, Inc.) pp 229-30

[25] Nagy A P 2011 Isogeometric design optimisation PhD dissertation, Delft University of Technology (available at: http://resolver.tudelft.nl/uuid:de6aef44-7d24-49aa-9485064c52a147fa)

[26] Hering S, Keunecke D and Niemz P 2012 Moisture-dependent orthotropic elasticity of beech wood Wood Sci. Technol. 46 927-38

[27] Niemz P, Ozyhar T, Hering S and Sonderegger W 2015 Moisture dependent physical-mechanical properties from beech wood in the main directions Pro Ligno 11 37-42

[28] Güntekin E, Aydin T Y and Niemz P 2016 Some orthotropic elastic properties of fagus orientalis as influenced by moisture content Wood Res. 61 95-104

[29] Schneider C A, Rasband W S and Eliceiri K W 2012 NIH Image to ImageJ: 25 years of image analysis Nat. Methods $9671-5$

[30] Seffen K A 2007 Hierarchical multi-stable shapes in mechanical memory metal Scr. Mater. 56 417-20

[31] Udani J P and Arrieta A F 2019 Analytical modeling of multi-sectioned bi-stable composites: stiffness variability and embeddability Compos. Struct. 216 228-39

[32] Yang H and Ma L 2020 1D to 3D multi-stable architected materials with zero Poisson's ratio and controllable thermal expansion Mater. Design 188 1-16

[33] Arrieta A F, Van Gemmeren V, Anderson A J and Weaver P M 2018 Dynamics and control of twisting bi-stable structures Smart Mater. Struct. 27025006 